\title{
The potential of dual-energy computed tomography for quantitative decomposition of soft tissues to water, protein and lipid in brachytherapy
}

\author{
Alexandr Malusek, M Karlsson, Maria Magnusson and Gudrun Alm Carlsson
}

\section{Linköping University Post Print}

N.B.: When citing this work, cite the original article.

Original Publication:

Alexandr Malusek, M Karlsson, Maria Magnusson and Gudrun Alm Carlsson, The potential of dual-energy computed tomography for quantitative decomposition of soft tissues to water, protein and lipid in brachytherapy, 2013, Physics in Medicine and Biology, (58), 4, 771-785. http://dx.doi.org/10.1088/0031-9155/58/4/771

Copyright: Institute of Physics http://www.iop.org/

Postprint available at: Linköping University Electronic Press http://urn.kb.se/resolve?urn=urn:nbn:se:liu:diva-89734 


\title{
The potential of dual-energy computed tomography for quantitative decomposition of soft tissues to water, protein and lipid in brachytherapy
}

\author{
A Malusek ${ }^{1}, \mathrm{M}$ Karlsson$^{1}, \mathrm{M}$ Magnusson $^{2}$ and G Alm Carlsson ${ }^{1}$ \\ ${ }^{1}$ Radiation Physics, Department of Medical and Health Sciences, Linköping \\ University, SE-581 85 Linköping, Sweden \\ ${ }^{2}$ Department of Electrical Engineering, Linköping University, SE-581 83 Linköping, \\ Sweden \\ E-mail: Alexandr.Malusek@liu.se
}

\begin{abstract}
Dosimetric accuracy of radiation treatment planning in brachytherapy depends on knowledge of tissue composition. It has been speculated that soft tissues can be decomposed to water, lipid and protein. The aim of our work is to evaluate the accuracy of such tissue decomposition. Selected abdominal soft tissues, whose average elemental compositions were taken from literature, were decomposed using dual energy computed tomography to water, lipid, and protein via the three-material decomposition method. The quality of the decomposition was assessed using relative differences between the (i) mass energy absorption and (ii) mass energy attenuation coefficients of the analyzed and approximated tissues. It was found that the relative differences were less than $2 \%$ for photon energies larger than $10 \mathrm{keV}$. The differences were notably smaller than the ones for water as the transport and dose scoring medium. The choice of the water, protein and lipid triplet resulted in negative elemental mass fractions for some analyzed tissues. As negative elemental mass fractions cannot be used in general purpose particle transport computer codes using the Monte Carlo method, other triplets should be used for the decomposition. These triplets may further improve the accuracy of the approximation as the differences were mainly caused by the lack of high- $Z$ materials in the water, protein and lipid triplet.
\end{abstract}

PACS numbers: 87.57.Q-, 87.57.nm

Keywords: DECT, tissue classification, brachytherapy

Submitted to: Phys. Med. Biol. 


\section{Introduction}

Dose calculation algorithms used in clinical treatment planning systems for brachytherapy (BT) are simplistic and derive the dose by superposing dose distributions from individual sources pre-calculated in unbounded water medium. The approach neglects effects of heterogeneities such as variations in tissue composition and density. Currently BT is developing fast towards introduction of sophisticated algorithms for imaged based dose calculation (Rivard et al 2009). These developments raise urgent demands for improved knowledge of atomic compositions of tissues in the individual patient (Beaulieu et al 2012). While dose calculations in external beam therapy depend mainly on electron densities due to the dominance of the Compton process, dose calculations in BT depend strongly on atomic number due to the influence of photoelectric absorption at the low energies of photons emitted from BT sources.

Attempts to perform tissue classification via computed tomography (CT) date back to the time when CT scanners first appeared in clinical practice. Alvarez and Macovski (1976) separated the linear attenuation coefficient (LAC) of each voxel into the photoelectric and Compton scattering components using approximative analytic formulas for the cross sections. The authors, however, did not tune the method for usage in radiation treatment. Radiation treatment with external beams has long been based on experimentally determined calibration curves relating relative electron densities to CT numbers obtained with single-energy CT scanners, see e.g. (Schneider, 1996). To improve the accuracy of tissue classification by utilizing the dual-energy CT scanners, Bazalova et al (2008a, 2008b) proposed a method based on the separation of the LAC to photoelectric and Compton scattering components according to Torikoshi et al (2003). The method allowed the determination of an effective $\mathrm{Z}$ and electron density of each voxel; this information was used for a more accurate classification. This principle was extended to BT with low energy photons by Landry et al (2010, 2011a, 2011b). The accuracy of effective $\mathrm{Z}$ numbers derived from dual-energy computed tomography (DECT) scans was investigated by Goodsitt et al (2011).

A different approach to tissue classification based on DECT is used in the so called three-material decomposition method, where a tissue is approximated by a mixture of three base materials. A decomposition to an average soft tissue, bone and iodine, or fat, soft tissue and iodine can be used for contrast agent detection (Petersilka et al 2008). Other suitable choices are calcium oxalate, cystine, and uric acid for the classification of kidney stones and gallstones (Graser et al 2008, Primak et al 2007, Scheffel et al 2007). Liu et al (2009) investigated the three-material decomposition method in phantom studies to determine the concentrations of hydroxyapatite in mixtures of water, hydroxyapatite and iron nitrate. They concluded that the validity of the method relied on using base materials with large differences in x-ray attenuation; scatter and other artifacts degraded the accuracy of CT numbers and affected the results negatively.

The introduction of DECT, where the CT scanner measures projection data obtained for two different tube voltages more or less simultaneously, was a major step in 
the improvement of accuracy of tissue classification as the scanning at several different tube voltages by single-energy CT scanners was strongly affected by motion artifacts. Another major step is expected from the introduction of spectral CT in the future. In this case the CT scanner analyzes energy imparted to detector elements by individual photons. Theoretical studies (Bornefalk 2012) indicate that the number of bases can be increased from 2 currently used in DECT to approximately 4 to 5 in spectral CT.

Measured elemental compositions of tissues were published for instance in (Woodard and White 1986), ICRP Publication 23 (ICRP 1975), and ICRU Report 46 (ICRU 1992). One approach to determine them was to calculate them from known elemental compositions of water, lipid, protein, carbohydrate, and ash, whose mass fractions were measured for each tissue. As water, lipid and protein form major part of soft tissues, it had been hypothesized that radiation treatment planning via CT can be based on the decomposition of soft tissues to those three components. The aim of this article

is to evaluate the accuracy of this approximation for selected abdominal soft tissues whose elemental composition was published in literature. As calcification may occur in some tumors, and the materials of the water, protein and lipid triplet do not contain any calcium, alternative approaches to material decomposition are also discussed. The selection of a suitable triplet is not a simple task. Thus another aim of the article is to present tools like a matrix formulation of the problem and graphical representations of the results; these tools can help in the development of complex tissue classification schemes based on DECT.

\section{Theory}

\subsection{Mixture rule}

In the independent atom approximation, the mass attenuation coefficient (MAC), $\mu / \rho$, of a mixture of $n$ components can be calculated as a sum of MACs, $(\mu / \rho)_{i}$, of individual components weighted over mass fractions $w_{i}$ as (Attix 1986)

$$
\mu / \rho=\sum_{i=1}^{n} w_{i}(\mu / \rho)_{i}
$$

For instance MACs of tissues in table 1 can be calculated from elemental mass fractions given in the table and EPDL (Cullen et al 1997) or XCOM (Hubbell and Seltzer, 2004) libraries containing photon cross section data, see further Section 3. For $n_{E}$ different photon energies $E_{1}, \ldots, E_{n_{E}}$, equation (1) leads to a set of $n_{E}$ equations, one equation for one energy. Matrix formulation of the problem is in the Appendix A.1.

The summation rule can also be used for linear attenuation coefficients (LACs). Using the fact that $\mu=\rho(\mu / \rho)$, where $\rho$ is the mass density of the material, equation (1) can be written as

$$
\mu=\rho \sum_{i=1}^{n} w_{i}\left(\frac{\mu}{\rho}\right)_{i}=\frac{m}{V} \sum_{i=1}^{n} \frac{m_{i}}{m} \frac{\mu_{i}}{m_{i} / V_{i}}=\sum_{i=1}^{n} \frac{V_{i}}{V} \mu_{i}=\sum_{i=1}^{n} v_{i} \mu_{i}
$$


where $\mu$ and $\mu_{i}$ are the LACs of the mixture and component $i$, respectively, $m$ and $V$ are the mass and volume, respectively, of the mixture, $m_{i}$ and $V_{i}$ are the mass and partial volume, respectively, of the $i$ th component, and $v_{i}$ is a volume fraction. It is important to realize that the symbols $m_{i}$ and $V_{i}$ in equation (2) denote the mass and partial volume of the $i$ th component both inside (the ratios $m_{i} / m$ and $v_{i} / v$ ) and outside (the ratio $m_{i} / V_{i}$ ) the mixture. Thus the derivation of equation (2) requires that initial masses and partial volumes of individual components are preserved when these components are added to a mixture. If these conditions are fulfilled then equations (1) and (2) are equivalent in the sense that one can be transformed to the other. In practice, however, the partial volumes are typically not preserved and therefore equations (1) and (2) lead to different results; more information about this issue is in sections Results and Appendix A.2. As in case of MACs, equation (2) leads to a set of $n_{E}$ equations for $n_{E}$ different photon energies $E_{1}, \ldots, E_{n_{E}}$, see the Appendix A.1.

\subsection{Decomposition and graphical representations}

2.2.1. $M A C$ and $L A C$ diagrams DECT allows several tissue classification schemes depending on which variables in equations (1) or (2) are known. As Monte Carlo codes for particle transport require both material composition and mass density for each voxel, the latter is also mentioned here. The most straightforward is a threematerial decomposition using MACs. This scheme, denoted as M3 in this article, is based on equation (1) where unknown variables are mass fractions $w_{1}, w_{2}$, and $w_{3}$. The normalization condition $w_{1}+w_{2}+w_{3}=1$ is equivalent to the statement that the mass of the mixture equals the sum of masses of all components. M3 is suitable for theoretical calculations, but its usage in CT is limited since CT scanners measure LACs and not MACs. Mass density, $\rho$, of the approximated mixture can be calculated from equation (A.9) if partial volumes are conserved.

For data from CT scanners, a three-material decomposition using LACs is more suitable. This scheme, denoted as L3 here, is based on equation (2) where the unknown variables are volume fractions $v_{1}, v_{2}$, and $v_{3}$. The normalization condition $v_{1}+v_{2}+v_{3}=1$ is equivalent to the statement that the volume of the mixture equals the sum of partial volumes of all components. Mass density, $\rho$, of the approximated mixture can be calculated from (A.10) if partial volumes are conserved.

Equations (1) and (2) can be presented in a graphical form. Let $L=\left(\mu\left(E_{1}\right), \mu\left(E_{2}\right)\right)$ and $L_{i}=\left(\mu_{i}\left(E_{1}\right), \mu_{i}\left(E_{2}\right)\right)$, where $1 \leq i \leq 3$, be points for LACs of the mixture and base components, respectively. Then equation (2) can be written as $L=L_{3}+$ $v_{1}\left(L_{1}-L_{3}\right)+v_{2}\left(L_{2}-L_{3}\right)$, i.e. the point $L$ is given by the vector addition rule which determines the volume fractions $v_{1}$ and $v_{2}$, see figure 1 . The volume fraction $v_{3}$ is given as $v_{3}=1-\left(v_{1}+v_{2}\right)$. It can be shown that all points inside the triangle with vertexes $L_{1}, L_{2}$ and $L_{3}$ have positive volume fractions. A point outside the triangle has at least one negative volume fraction. Similar diagram can be plotted for MACs. The unknown linear coefficients are then the mass fractions $w_{1}, w_{2}$ and $w_{3}$. 


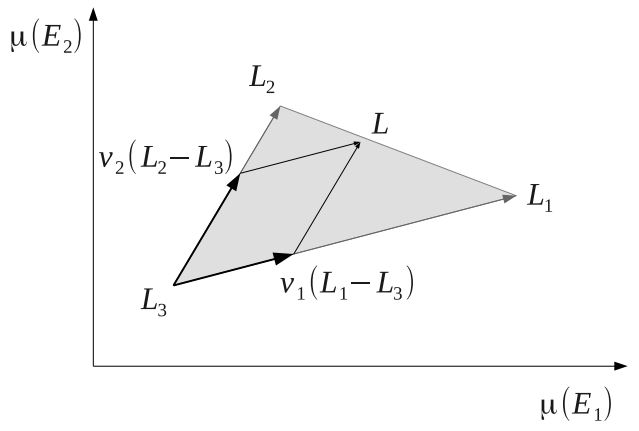

(a)

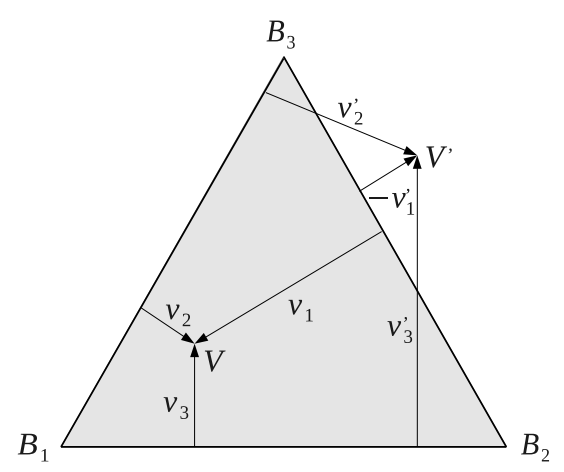

Figure 1. (a) LAC diagram. The volume fractions $v_{1}$ and $v_{2}$ are given by the condition that the point $L$ is obtained by adding vectors $v_{1}\left(L_{1}-L_{3}\right)$ and $v_{2}\left(L_{2}-L_{3}\right)$ to the point $L_{3}$, i.e. $L=L_{3}+v_{1}\left(L_{1}-L_{3}\right)+v_{2}\left(L_{2}-L_{3}\right)$. (b) Ternary plot. The volume fractions $v_{1}, v_{2}$ and $v_{3}$ are measured as distances from bases of the triangle relative to the height of the triangle. Points outside the triangle have at least one negative volume fraction.

2.2.2. Ternary plots $\mathrm{LAC}$ and MAC diagrams are difficult to read when the triangles like $L_{1}, L_{2}$ and $L_{3}$ in figure 1(a) are very flat. In this case a better form of presentation is a ternary plot, see figure $1(\mathrm{~b})$. The volume fractions $v_{1}, v_{2}$ and $v_{3}$ are measured as distances from bases of the equilateral triangle relative to the height of the triangle, see e.g. (Aitchison 1986). Ternary plots are typically used in physical sciences to show the compositions of systems composed of three species and thus negative coordinates are not considered. The condition $v_{1}+v_{2}+v_{3}=1$ follows from the fact that the area of the equilateral triangle with vertexes $\left(B_{1}, B_{2}, B_{3}\right)$ equals the sum of areas of triangles with vertexes $\left(B_{1}, B_{2}, V\right),\left(B_{2}, B_{3}, V\right)$ and $\left(B_{3}, B_{1}, V\right)$. For a point $V^{\prime}$ outside the equilateral triangle, the area of the $\left(B_{3}, B_{2}, V^{\prime}\right)$ triangle must be taken with a negative sign, hence the coordinate $v_{1}^{\prime}$ is negative.

2.2.3. Two-material decomposition Contrary to conservation of mass, conservation of volume fractions is not generally valid and thus the calculated mass density of the mixture (Appendix A.2) may be noticeably biased, see section 5 for more information. A two-material decomposition may be used in this case. Of practical importance is the scheme denoted as M2 in this paper, where the unknown variables are the density of the mixture $\rho$ and the mass fractions $w_{1}$ and $w_{2}$. The normalization condition is $w_{1}+w_{2}=1$. Using the notation $M=\left(\mu\left(E_{1}\right) / \rho, \mu\left(E_{2}\right) / \rho\right)$ and $M_{i}=\left(\mu_{i}\left(E_{1}\right) / \rho_{i}, \mu_{i}\left(E_{2}\right) / \rho_{i}\right)$ for the MACs of the mixture and base components, respectively, equation (1) can be written as $M=q L / \rho_{\mathrm{w}}=M_{2}+w_{1}\left(M_{1}-M_{2}\right)$, where $q=\rho_{\mathrm{w}} / \rho$ is a dimensionless parameter. The density $\rho_{\mathrm{w}}$ can be set to any value, for instance $1 \mathrm{~g} / \mathrm{cm}^{3}$. The mass fraction $w_{1}$ is then given by the intersection of the line connecting points $M_{2}$ and $M_{1}$ with a line connecting the origin and the point $L / \rho_{\mathrm{w}}$, see figure 2 . 


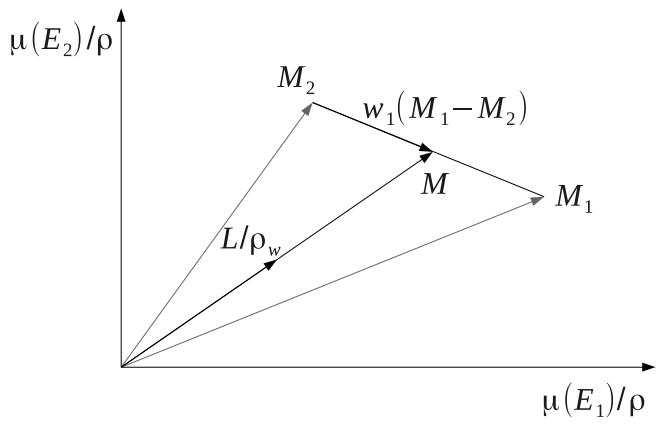

Figure 2. MAC diagram for the M2 method. The mass fraction $w_{1}$ is given by the intersection of the line $M_{2}+w\left(M_{1}-M_{2}\right)$ with the ray $q L / \rho_{\mathrm{w}}$, where $w$ and $q>0$ are parameters.

2.2.4. Material decomposition from measured CT numbers LACs of base materials can be calculated from tabulated cross section data or measured using e.g. a CT scanner. In the latter case, the LAC is typically converted to a CT number defined as $H=1000\left(\mu / \mu_{\mathrm{w}}-1\right)$, where $\mu$ and $\mu_{\mathrm{w}}$ are LACs of the imaged object and water, respectively. The aim of this definition is to make the CT number less dependent on photon energy. Equation (2) can be written as

$$
H=\sum_{i=1}^{n} v_{i} H_{i}
$$

where $H$ is the $\mathrm{CT}$ number of mixture and $H_{i}$ are $\mathrm{CT}$ numbers of components of the mixture. In this case, the volume fractions $v_{i}$ must fulfill the normalization condition $v_{1}+v_{2}+v_{3}=1$. Values of $H_{i}$ may be measured by the $\mathrm{CT}$ scanner for 2 different x-ray tube voltages and the resulting system of equations can be used for a tissue decomposition using the schemes described above.

\section{Methods}

Selected soft tissues from the abdominal region (table 1) were decomposed to water, protein and lipid (table 2) via equations (A.7) and (A.8), which are based on the L3 and M3 methods, respectively, described in section 2.2. Constants used for the calculation of matrix dimensions in these equations are in table 3. Mass densities and elemental compositions of soft tissues and base materials were taken from ICRU Report 44 (1989), ICRU Report 46 (1992) and (Woodard and White, 1986). MACs of all materials were calculated from photon cross section data in the EPDL97 data library by summing macroscopic cross sections of photoelectric effect, coherent scattering and incoherent scattering. MACs were taken at photon energies of $E_{1}=54.6 \mathrm{keV}$ and $E_{2}=94.0 \mathrm{keV}$. These two energies were calculated as energy-fluence weighted mean energies of energy spectra of the Siemens Somatom Definition CT scanner for x-ray tube voltages of 80 and $140 \mathrm{kV}$; the latter spectrum was for the configuration with an additional tin filter. 
Quantitative decomposition of tissues via DECT

Table 1. Published mass densities, $\rho$, and elemental mass fractions, $w^{\mathrm{e}}$, of selected abdominal soft tissues. Abbreviated names: adipose tissue, adrenal gland, gallbladder bile, gastrointestinal tract, skeletal muscle, small intestine, urinary bladder empty and urinary bladder filled.

\begin{tabular}{|c|c|c|c|c|c|c|c|c|c|c|c|c|}
\hline \multirow[b]{2}{*}{ tissue } & \multirow{2}{*}{$\begin{array}{l}\rho \\
\left(\mathrm{g} / \mathrm{cm}^{3}\right)\end{array}$} & \multicolumn{11}{|c|}{ elemental mass fraction, $w^{\mathrm{e}}(\%)$} \\
\hline & & $\mathrm{H}$ & $\mathrm{C}$ & $\mathrm{N}$ & $\mathrm{O}$ & $\mathrm{Na}$ & $\mathrm{S}$ & $\mathrm{Cl}$ & $\mathrm{P}$ & $\mathrm{K}$ & $\mathrm{Fe}$ & $\mathrm{Ca}$ \\
\hline adipose t. $1^{b}$ & 0.97 & 11.2 & 51.7 & 1.3 & 35.5 & 0.1 & 0.1 & 0.1 & 0.0 & 0.0 & 0.0 & 0.0 \\
\hline adipose t. $2^{b}$ & 0.95 & 11.4 & 59.8 & 0.7 & 27.8 & 0.1 & 0.1 & 0.1 & 0.0 & 0.0 & 0.0 & 0.0 \\
\hline adipose t. $3^{b}$ & 0.93 & 11.6 & 68.1 & 0.2 & 19.8 & 0.1 & 0.2 & 0.1 & 0.0 & 0.0 & 0.0 & 0.0 \\
\hline adrenal g. ${ }^{c}$ & 1.03 & 10.6 & 28.4 & 2.6 & 57.8 & 0.0 & 0.2 & 0.2 & 0.1 & 0.1 & 0.0 & 0.0 \\
\hline g. bile ${ }^{c}$ & 1.03 & 10.8 & 6.1 & 0.1 & 82.2 & 0.4 & 0.0 & 0.4 & 0.0 & 0.0 & 0.0 & 0.0 \\
\hline blood $^{a}$ & 1.06 & 10.2 & 11.0 & 3.3 & 74.5 & 0.1 & 0.2 & 0.3 & 0.1 & 0.2 & 0.1 & 0.0 \\
\hline g.i. $\operatorname{tract}^{a}$ & 1.03 & 10.6 & 11.5 & 2.2 & 75.1 & 0.1 & 0.1 & 0.2 & 0.1 & 0.1 & 0.0 & 0.0 \\
\hline kidney $1^{c}$ & 1.05 & 10.2 & 16.0 & 3.4 & 69.3 & 0.2 & 0.2 & 0.2 & 0.2 & 0.2 & 0.0 & 0.1 \\
\hline kidney $2^{c}$ & 1.05 & 10.3 & 13.2 & 3.0 & 72.4 & 0.2 & 0.2 & 0.2 & 0.2 & 0.2 & 0.0 & 0.1 \\
\hline kidney $3^{c}$ & 1.05 & 10.4 & 10.6 & 2.7 & 75.2 & 0.2 & 0.2 & 0.2 & 0.2 & 0.2 & 0.0 & 0.1 \\
\hline liver $1^{c}$ & 1.05 & 10.3 & 15.6 & 2.7 & 70.1 & 0.2 & 0.3 & 0.2 & 0.3 & 0.3 & 0.0 & 0.0 \\
\hline liver $2^{c}$ & 1.06 & 10.2 & 13.9 & 3.0 & 71.6 & 0.2 & 0.3 & 0.2 & 0.3 & 0.3 & 0.0 & 0.0 \\
\hline liver $3^{c}$ & 1.07 & 10.1 & 12.6 & 3.3 & 72.7 & 0.2 & 0.3 & 0.2 & 0.3 & 0.3 & 0.0 & 0.0 \\
\hline $\operatorname{lymph}^{a}$ & 1.03 & 10.8 & 4.1 & 1.1 & 83.2 & 0.3 & 0.1 & 0.4 & 0.0 & 0.0 & 0.0 & 0.0 \\
\hline s. muscle $1^{c}$ & 1.05 & 10.1 & 17.1 & 3.6 & 68.1 & 0.1 & 0.3 & 0.1 & 0.0 & 0.4 & 0.0 & 0.0 \\
\hline s. muscle $2^{c}$ & 1.05 & 10.2 & 14.3 & 3.4 & 71.0 & 0.1 & 0.3 & 0.1 & 0.0 & 0.4 & 0.0 & 0.0 \\
\hline s. muscle $3^{c}$ & 1.05 & 10.2 & 11.2 & 3.0 & 74.5 & 0.1 & 0.3 & 0.1 & 0.0 & 0.4 & 0.0 & 0.0 \\
\hline ovary $^{a}$ & 1.05 & 10.5 & 9.3 & 2.4 & 76.8 & 0.2 & 0.2 & 0.2 & 0.2 & 0.2 & 0.0 & 0.0 \\
\hline pancreas $^{a}$ & 1.04 & 10.6 & 16.9 & 2.2 & 69.4 & 0.2 & 0.1 & 0.2 & 0.2 & 0.2 & 0.0 & 0.0 \\
\hline prostate $^{c}$ & 1.04 & 10.5 & 8.9 & 2.5 & 77.4 & 0.2 & 0.2 & 0.0 & 0.1 & 0.2 & 0.0 & 0.0 \\
\hline $\operatorname{skin} 1^{c}$ & 1.09 & 10.0 & 25.0 & 4.6 & 59.4 & 0.2 & 0.3 & 0.3 & 0.1 & 0.1 & 0.0 & 0.0 \\
\hline skin $2^{c}$ & 1.09 & 10.0 & 20.4 & 4.2 & 64.5 & 0.2 & 0.2 & 0.3 & 0.1 & 0.1 & 0.0 & 0.0 \\
\hline $\operatorname{skin} 3^{c}$ & 1.09 & 10.1 & 15.8 & 3.7 & 69.5 & 0.2 & 0.2 & 0.3 & 0.1 & 0.1 & 0.0 & 0.0 \\
\hline s. intestine ${ }^{c}$ & 1.03 & 10.6 & 11.5 & 2.2 & 75.1 & 0.1 & 0.1 & 0.2 & 0.1 & 0.1 & 0.0 & 0.0 \\
\hline spleen $^{a}$ & 1.06 & 10.3 & 11.3 & 3.2 & 74.1 & 0.1 & 0.2 & 0.2 & 0.3 & 0.3 & 0.0 & 0.0 \\
\hline stomach $^{c}$ & 1.05 & 10.4 & 13.9 & 2.9 & 72.1 & 0.1 & 0.2 & 0.1 & 0.1 & 0.2 & 0.0 & 0.0 \\
\hline u. bladder e. ${ }^{b}$ & 1.04 & 10.5 & 9.6 & 2.6 & 76.1 & 0.2 & 0.2 & 0.3 & 0.2 & 0.3 & 0.0 & 0.0 \\
\hline u. bladder $\mathrm{f}^{b}$ & 1.03 & 10.8 & 3.5 & 1.5 & 83.0 & 0.3 & 0.1 & 0.5 & 0.1 & 0.2 & 0.0 & 0.0 \\
\hline
\end{tabular}

${ }^{a}$ ICRU Report 44 (1989)

${ }^{b}$ ICRU Report 46 (1992)

${ }^{c}$ Woodard and White (1986) 
Table 2. Mass densities, $\rho$, and elemental compositions of the base materials. Mass fractions are given in $\%$.

\begin{tabular}{llrrrrr}
\hline & & \multicolumn{5}{c}{ elemental mass fr., $w^{\mathrm{e}}(\%)$} \\
\cline { 2 - 6 } material & $\left(\mathrm{g} / \mathrm{cm}^{3}\right)$ & $\mathrm{H}$ & $\mathrm{C}$ & $\mathrm{N}$ & $\mathrm{O}$ & $\mathrm{S}$ \\
\hline lipid & 0.92 & 11.8 & 77.3 & 0.0 & 10.9 & 0.0 \\
protein & 1.35 & 6.6 & 53.4 & 17.0 & 22.0 & 1.0 \\
water & 1.00 & 11.2 & 0.0 & 0.0 & 88.8 & 0.0 \\
\hline
\end{tabular}

Table 3. Constants defining dimensions used in the matrix formulation.

\begin{tabular}{lll}
\hline symbol & value & description \\
\hline$n_{E}$ & 2 & number of energies at which $\mu$ is evaluated \\
$n_{b}$ & 3 & number of base materials \\
$n_{e}$ & 11 & number of elements $(\mathrm{H}, \mathrm{C}, \ldots)$ in tissue composition tables \\
$n_{v}$ & 28 & number of simultaneously analyzed voxels. \\
\hline
\end{tabular}

Both spectra were provided by Siemens under a non-disclosure agreement. LACs were calculated from MACs as $\mu=(\mu / \rho) \rho$, where mass densities were taken from tables 1 and 2 .

Elemental mass fractions $W_{\left(n_{E} \times n_{e}\right)}^{\mathrm{e}}$ corresponding to volume fractions $V_{\left(n_{b} \times n_{v}\right)}$ obtained in the previous stage via the L3 method (equation (A.8)) were calculated in two steps. First, mass fractions $W_{\left(n_{b} \times n_{v}\right)}$ of the water, lipid, protein triplet for all analyzed tissues were calculated from the volume fractions as

$$
W_{\left(n_{b} \times n_{v}\right)}=\rho_{\left(n_{b} \times n_{b}\right)} V_{\left(n_{b} \times n_{v}\right)}\left(1 / \rho^{\mathrm{a}}\right)_{\left(n_{v} \times n_{v}\right)},
$$

where $\rho_{\left(n_{b} \times n_{b}\right)}=\operatorname{diag}\left(\rho_{1}, \ldots, \rho_{n_{b}}\right)$ is a diagonal matrix containing mass densities of base materials, and $\left(1 / \rho^{\mathrm{a}}\right)_{\left(n_{v} \times n_{v}\right)}=\operatorname{diag}\left(1 / \rho_{1}^{\mathrm{a}}, \ldots, 1 / \rho_{n_{v}}^{\mathrm{a}}\right)$ is a diagonal matrix containing reciprocal values of mass densities of the approximated tissues calculated from equation (A.10). In the second step, the elemental mass fractions $W_{\left(n_{e} \times n_{v}\right)}^{\mathrm{e}}$ were calculated as

$$
W_{\left(n_{e} \times n_{v}\right)}^{\mathrm{e}}=W_{\left(n_{e} \times n_{b}\right)}^{\mathrm{e}} W_{\left(n_{b} \times n_{v}\right)},
$$

where $W_{\left(n_{e} \times n_{b}\right)}^{\mathrm{e}}$ are elemental mass fractions of the water, lipid, protein triplet in table 2 and $W_{\left(n_{b} \times n_{v}\right)}$ are mass fractions calculated in the previous step. To avoid a misunderstanding, we recall that mass fractions $W_{\left(n_{b} \times n_{v}\right)}$ can also be calculated via the M3 method from equation (A.7).

\subsection{Quality of the decomposition}

Ideally a tissue would be decomposed into mass fractions of all its elements. Most tissues, however, consist of up to 10 elements (table 1) and so such decomposition is impossible when only two tube voltages are used. Thus the presented decomposition methods are approximate only. For instance the L3 method determines volume fractions 
of base materials so that the LAC of the approximated mixture equals the LAC of the analyzed tissue at two selected photon energies $E_{1}$ and $E_{2}$. At other photon energies, however, this equality may not hold since the approximated tissues do not contain elements like $\mathrm{Na}, \mathrm{Cl}, \mathrm{P}, \mathrm{K}$ and $\mathrm{Fe}$ in case of the water, lipid and protein triple. These high- $Z$ elements increase LACs of the analyzed tissues especially at low photon energies where the photoelectric effect dominates. At the selected photon energies $E_{1}$ and $E_{2}$, the three-material decomposition method compensates for the increase in LAC by using unrealistic (e.g., negative) volume fractions; this issue is further discussed in Section 5. The two-material decomposition method is more robust as it also adjusts the mass density of the approximated mixture to make the LACs equal. At low photon energies, however, this compensation may become insufficient for both the two-material and the three-material decomposition methods. To address this issue, two measures of the quality of decomposition are considered in this paper: the difference between the (i) MACs and (ii) mass energy absorption coefficients of the analyzed and approximated tissues. The first one is important for image reconstruction in CT and photon transport simulations. The second one is important for the calculation of absorbed doses via the kerma approximation. MACs of approximated tissues, $\mu^{\mathrm{a}}(E) / \rho^{\mathrm{a}}$, were calculated via the mixture rule as

$$
\mu^{\mathrm{a}}(E) / \rho^{\mathrm{a}}=\sum_{i=1}^{n_{e}} w_{i}^{\mathrm{e}} \mu_{i}^{\mathrm{e}}(E) / \rho,
$$

where $w_{i}^{\mathrm{e}}$ is the mass fraction of element $i$ in the approximated mixture, $\mu_{i}^{\mathrm{e}}(E) / \rho$ is the MAC for element $i$, and $n_{e}$ is the number of elements in the mixture. Mass energy absorption coefficients, $\mu_{\mathrm{en}}^{\mathrm{a}}(E) / \rho^{\mathrm{a}}$, were calculated as

$$
\mu_{\mathrm{en}}^{\mathrm{a}}(E) / \rho^{\mathrm{a}}=\sum_{i=1}^{n_{e}} w_{i} \mu_{\mathrm{en}, i}^{\mathrm{e}}(E) / \rho,
$$

where $\mu_{\mathrm{en}, i}^{\mathrm{e}}(E) / \rho$ is the mass energy absorption coefficient for element $i$. Other symbols have the same meaning as in equation (6). The values of $\mu_{i}^{\mathrm{e}}(E) / \rho$ and $\mu_{\mathrm{en}, i}^{\mathrm{e}}(E) / \rho$ were taken from (Hubbell and Seltzer 2004). Elements with negative mass fractions (cf. table 4) contributed negative values to both the MAC and the mass energy absorption coefficient.

\section{Results}

\subsection{Decomposition to water, protein and lipid: mass and volume fractions}

MAC and LAC diagrams for tissues in table 1 are in figure 3. Only adrenal gland, skin 1 and adipose tissues 1, 2, and 3 are inside the triangle in figure 3(a). Thus only those tissues can be decomposed to positive mass fractions of water, protein and lipid. Similarly, only the adrenal gland and adipose tissues 1 and 2 are inside the triangle in figure 3(b), and thus only those tissues can be decomposed to positive volume fractions of the same triplet. The fact that the mass fraction of protein in adipose tissue 3 is 


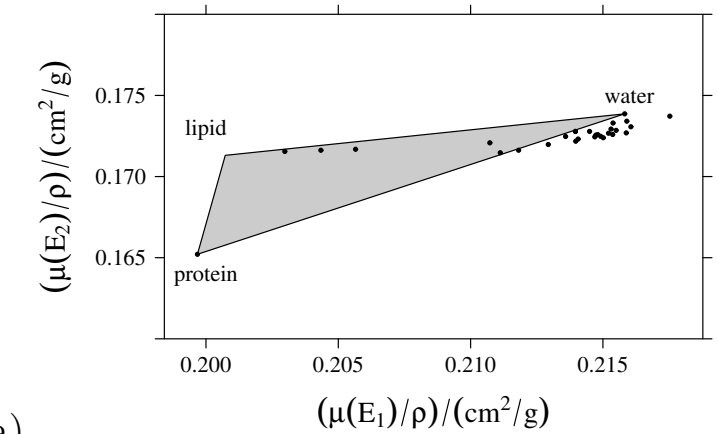

(a)

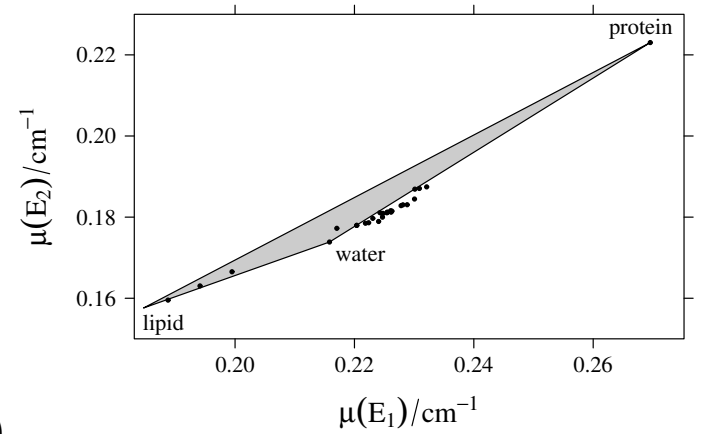

Figure 3. (a) Mass attenuation coefficients, $\mu(E) / \rho$, and (b) linear attenuation coefficients, $\mu(E)$, taken at energies $E_{1}=54.6 \mathrm{keV}$ and $E_{2}=94.2 \mathrm{keV}$ for abdominal soft tissues in table 1. Triangles for the water, protein and lipid triplet are also shown.

positive and corresponding volume fraction is negative indicates that the conservation of partial volumes is not fulfilled for this tissue.

Volume fractions $v_{1}, v_{2}$ and $v_{3}$ of the water, protein and lipid triplet calculated from equation (A.8) for all tissues in table 1 are in table 4. Ternary plot of the resulting volume fractions is in figure $4(\mathrm{~b})$. Table 4 also contains mass densities of the approximated

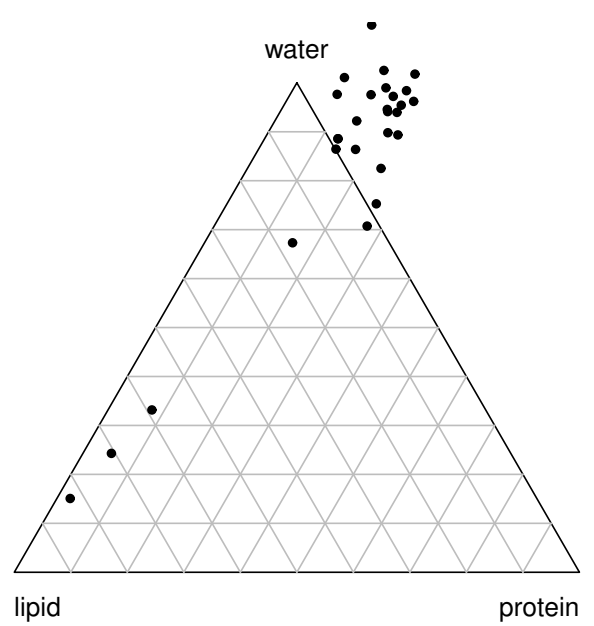

(a) lipid protein

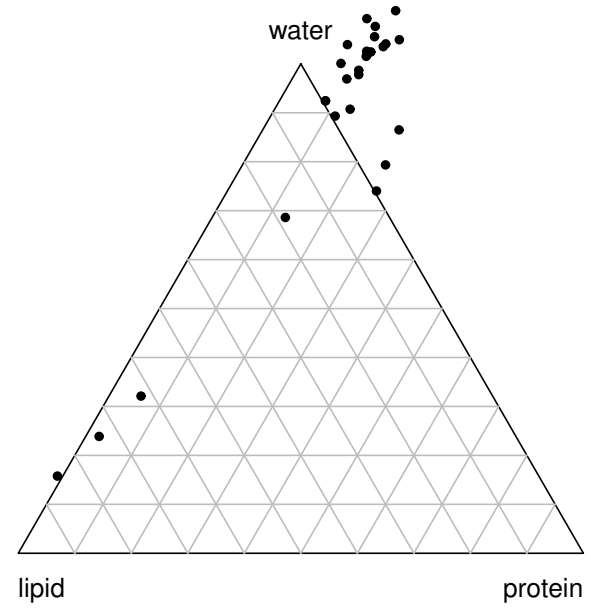

lipid

Figure 4. Ternary plots of (a) mass fractions and (b) volume fractions of selected abdominal tissues decomposed to water, lipid and protein.

mixtures calculated from equation (A.10), and elemental mass fractions calculated from the volume fractions via equations (4) and (5). These elemental mass fractions of the approximated mixtures can be compared to the elemental mass fractions of analyzed tissues in table 1 . Note that the volume fraction of lipid in prostate was negative, but all elemental mass fractions were positive for this tissue. For liver, however, the negative volume fraction of lipid resulted in negative elemental mass fraction of carbon. The issue with negative mass and volume fractions is further discussed in section 5 .

Mass fractions of water, protein and lipid in analyzed tissues calculated via the 
M3 method from equation (A.7) are in figure 4(a). The similarity between figures 4(a) and 4(b) is caused by the fact that densities of base materials in table 2 were close to $1 \mathrm{~g} / \mathrm{cm}^{3}$. Thus the ratios $\rho_{i} / \rho$, where $\rho_{i}$ and $\rho$ are the densities of component $i$ and the mixture, respectively, were close to 1 ; these ratios convert volume fractions to mass fractions.

A large difference between results obtained via the L3 and M3 methods may appear if volume fractions are not preserved. As mass densities of analyzed tissues in table 1 are almost the same as calculated mass densities of approximated mixtures in table 4, it is reasonable to expect that the non-conservation of partial volumes introduced small differences only.

\subsection{Quality of the decomposition}

To assess the quality of the tissue decomposition from the point of view of radiation treatment planning, mass energy absorption coefficients and mass attenuation coefficients of approximated tissues were compared to true values. Relative differences $\delta_{\mathrm{en}}(E)=\left(\mu_{\mathrm{en}}^{\mathrm{a}}(E) / \rho^{\mathrm{a}}-\mu_{\mathrm{en}}(E) / \rho\right) /\left(\mu_{\mathrm{en}}(E) / \rho\right)$ between mass energy absorption coefficients of analyzed tissues, $\mu_{\mathrm{en}}(E) / \rho$, and approximated mixtures, $\mu_{\mathrm{en}}^{\mathrm{a}}(E) / \rho^{\mathrm{a}}$, calculated via the L3 method are in figure 5(a). The relative differences were less than

mass energy absorption coefficient

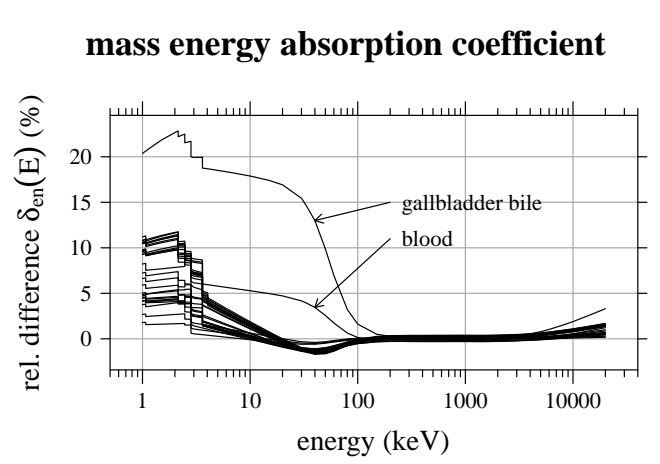

(a) (b)

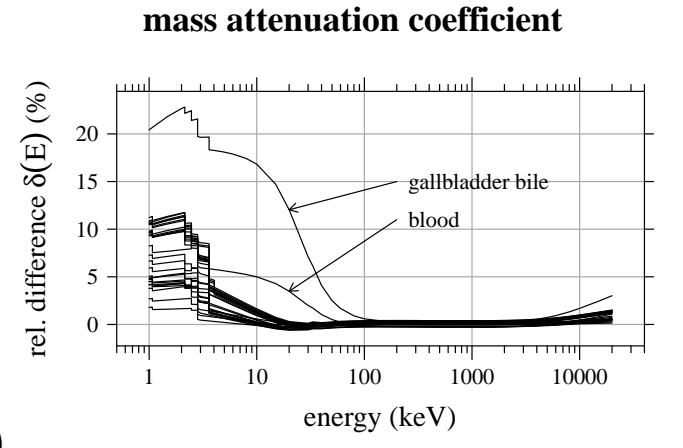

Figure 5. (a) Relative difference $\delta_{\text {en }}(E)$ between the mass energy absorption coefficients of approximated mixtures (table 4) and tabulated tissues (table 1) as a function of photon energy for all analyzed tissues. (b) Relative difference $\delta(E)$ between the mass attenuation coefficients of the approximated mixtures and analyzed tissues as a function of photon energy for all analyzed tissues.

$2 \%$ for photon energies larger than $10 \mathrm{keV}$ for most tissues. The notable exceptions were gallbladder bile and blood with relative differences of $18 \%$ and $5 \%$, respectively, at $10 \mathrm{keV}$. These tissues contain high-Z elements like $\mathrm{Cl}$ or Fe which are not in the base triplet. Relative differences $\delta(E)=\left(\mu^{\mathrm{a}}(E) / \rho^{\mathrm{a}}-\mu(E) / \rho\right) /(\mu(E) / \rho)$ between mass attenuation coefficients of analyzed tissues, $\mu(E) / \rho$, and approximated mixtures, $\mu^{\mathrm{a}}(E) / \rho^{\mathrm{a}}$, calculated via the L3 method are in figure $5(\mathrm{~b})$. The results were similar to those in figure 5(a). These results demonstrate that the water, lipid and protein triplet approximated most of the abdominal soft tissues well. It is reasonable to expect 
the existence of triplets providing even better approximations as the discrepancies were mainly caused by the lack of high-Z elements in the water, lipid and protein triplet. The addition of suitable high-Z elements to one or more base materials may also result in positive elemental mass fractions.

\subsection{Comparison with the all-water assumption}

To demonstrate the usability of the three-material decomposition in comparison with the hitherto used all-water assumption, figure $6(\mathrm{a})$ shows relative differences $\delta_{\mathrm{en}}^{\mathrm{w}}(E)=$ $\left(\mu_{\mathrm{en}}^{\mathrm{w}}(E) / \rho-\mu_{\mathrm{en}}(E) / \rho\right) /\left(\mu_{\mathrm{en}}(E) / \rho\right)$ between mass energy absorption coefficients of water, $\mu_{\mathrm{en}}^{\mathrm{w}} / \rho$, and analyzed tissues, $\mu_{\mathrm{en}} / \rho$. These relative differences were much larger than those in figure 5(a). The largest ones at $10 \mathrm{keV}$ were for adipose tissues 3, 2, and 1, gallbladder bile, and adrenal gland (in this order). Figure $6(\mathrm{~b})$ shows relative differences $\delta^{\mathrm{w}}(E)=\left(\mu^{\mathrm{w}}(E) / \rho^{\mathrm{w}}-\mu(E) / \rho\right) /(\mu(E) / \rho)$ between mass attenuation coefficients of water, $\mu^{\mathrm{w}} / \rho^{\mathrm{w}}$, and analyzed tissues, $\mu / \rho$. The differences were largest for the same tissues as in figure $6(\mathrm{a})$. Figure 6 clearly demonstrates that the radiation treatment planning using

\section{mass energy absorption coefficient}

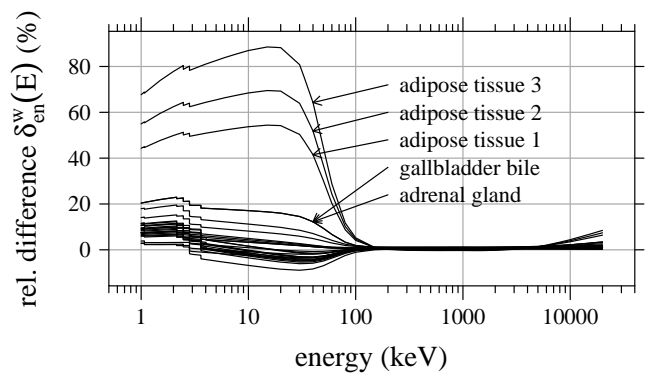

(a)

\section{mass attenuation coefficient}

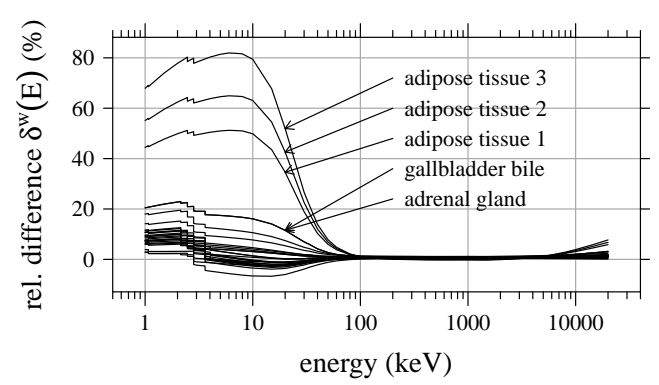

Figure 6. (a) Relative difference $\delta_{\text {en }}^{\mathrm{w}}(E)$ between the mass energy absorption coefficients of water and tabulated tissues (table 1) as a function of photon energy for all analyzed tissues. (b) Relative difference $\delta^{\mathrm{w}}(E)$ between the mass attenuation coefficients of the water and tabulated tissues as a function of photon energy for all analyzed tissues.

water as transport and dose scoring medium may lead to notable errors for photons with energies lower than $100 \mathrm{keV}$.

\section{Discussion}

\subsection{Accuracy of the three-material decomposition for use in transport codes}

The summation rule, equation (1), is valid if effects of molecular binding and crystal structure on photon cross sections can be neglected. In this respect the M3 method is more fundamental than the L3 method, which was derived under the assumption that volume fractions of individual components are preserved in the mixture. As the latter is not fulfilled in general, the volume fractions have been viewed as mere linear 
Quantitative decomposition of tissues via DECT

Table 4. Calculated attributes of approximated mixtures obtained from tissues in table 1 via the L3 method: mass density, $\rho^{\mathrm{a}}$, volume fractions, $v$, of the water, lipid and protein triplet, and corresponding elemental mass fractions, $w^{\mathrm{e}}$, of $\mathrm{H}, \mathrm{C}, \mathrm{N}, \mathrm{O}$ and S.

\begin{tabular}{|c|c|c|c|c|c|c|c|c|c|}
\hline \multirow[b]{2}{*}{ tissue } & \multirow{2}{*}{$\begin{array}{l}\rho^{\mathrm{a}} \\
\left(\mathrm{g} / \mathrm{cm}^{3}\right)\end{array}$} & \multicolumn{5}{|c|}{ elemental mass fr., $w^{\mathrm{e}}(\%)$} & \multicolumn{3}{|c|}{ volume fr., $v(\%)$} \\
\hline & & $\mathrm{H}$ & $\mathrm{C}$ & $\mathrm{N}$ & $\mathrm{O}$ & S & water & lipid & protein \\
\hline adipose t. 1 & 0.970 & 11.2 & 49.8 & 1.3 & 37.6 & 0.1 & 32.1 & 62.2 & 5.7 \\
\hline adipose t. 2 & 0.949 & 11.5 & 57.1 & 0.6 & 30.9 & 0.0 & 23.9 & 73.8 & 2.4 \\
\hline adipose t. 3 & 0.928 & 11.8 & 64.5 & -0.2 & 24.0 & -0.0 & 17.9 & 83.5 & -1.4 \\
\hline adrenal g. & 1.031 & 10.5 & 21.8 & 2.9 & 64.6 & 0.2 & 68.6 & 18.4 & 13.0 \\
\hline g. bile & 1.030 & 10.7 & 0.0 & 1.6 & 87.6 & 0.1 & 100.1 & -7.1 & 7.1 \\
\hline blood & 1.057 & 10.4 & -7.2 & 2.5 & 94.2 & 0.1 & 110.9 & -22.2 & 11.3 \\
\hline g.i. tract & 1.029 & 10.7 & 5.3 & 1.8 & 82.1 & 0.1 & 92.4 & -0.6 & 8.1 \\
\hline kidney 1 & 1.047 & 10.5 & 1.6 & 2.5 & 85.3 & 0.1 & 97.8 & -9.1 & 11.3 \\
\hline kidney 2 & 1.048 & 10.5 & -1.2 & 2.4 & 88.2 & 0.1 & 101.9 & -12.6 & 10.8 \\
\hline kidney 3 & 1.049 & 10.5 & -3.7 & 2.2 & 90.8 & 0.1 & 105.5 & -15.8 & 10.3 \\
\hline liver 1 & 1.048 & 10.5 & -0.9 & 2.4 & 87.9 & 0.1 & 101.5 & -12.3 & 10.8 \\
\hline liver 2 & 1.058 & 10.4 & -2.2 & 2.8 & 88.9 & 0.2 & 103.5 & -16.3 & 12.8 \\
\hline liver 3 & 1.068 & 10.2 & -3.1 & 3.2 & 89.5 & 0.2 & 104.9 & -19.9 & 15.0 \\
\hline lymph & 1.030 & 10.8 & -2.6 & 1.4 & 90.4 & 0.1 & 103.9 & -10.2 & 6.3 \\
\hline s. muscle 1 & 1.046 & 10.5 & 1.0 & 2.4 & 86.0 & 0.1 & 95.2 & -6.9 & 11.7 \\
\hline s. muscle 2 & 1.047 & 10.5 & -1.6 & 2.3 & 88.7 & 0.1 & 99.1 & -10.2 & 11.2 \\
\hline s. muscle 3 & 1.046 & 10.6 & -5.1 & 2.0 & 92.4 & 0.1 & 104.1 & -14.3 & 10.1 \\
\hline ovary & 1.050 & 10.5 & -1.5 & 2.4 & 88.5 & 0.1 & 102.4 & -13.6 & 11.2 \\
\hline pancreas & 1.041 & 10.5 & 7.4 & 2.5 & 79.4 & 0.1 & 89.3 & -0.7 & 11.4 \\
\hline prostate & 1.039 & 10.6 & 2.2 & 2.1 & 84.9 & 0.1 & 96.9 & -6.6 & 9.7 \\
\hline skin 1 & 1.093 & 9.7 & 17.1 & 5.5 & 67.3 & 0.3 & 74.0 & -0.4 & 26.4 \\
\hline skin 2 & 1.092 & 9.7 & 13.7 & 5.3 & 71.0 & 0.3 & 79.3 & -4.7 & 25.3 \\
\hline skin 3 & 1.093 & 9.8 & 9.0 & 5.1 & 75.9 & 0.3 & 86.5 & -10.6 & 24.1 \\
\hline s. intestine & 1.029 & 10.7 & 5.3 & 1.8 & 82.1 & 0.1 & 92.4 & -0.6 & 8.1 \\
\hline spleen & 1.059 & 10.3 & -2.6 & 2.8 & 89.3 & 0.2 & 104.1 & -17.1 & 13.0 \\
\hline stomach & 1.050 & 10.4 & 6.4 & 2.9 & 80.1 & 0.2 & 90.7 & -4.1 & 13.4 \\
\hline u. bladder e. & 1.038 & 10.7 & -6.3 & 1.6 & 93.9 & 0.1 & 109.2 & -16.3 & 7.1 \\
\hline u. bladder f. & 1.029 & 10.9 & -11.4 & 0.8 & 99.6 & 0.0 & 116.5 & -20.1 & 3.6 \\
\hline
\end{tabular}


coefficients in equation (2) without any deeper physical meaning e.g. by Liu et al (2009). For mixtures of soft tissues the assumption $1=v_{1}+v_{2}+v_{3}$ does not lead to large discrepancies in calculated mass densities; the largest relative difference $\left|\rho^{\mathrm{a}}-\rho\right| / \rho$ between calculated, $\rho^{\mathrm{a}}$, and tabulated, $\rho$, mass densities in tables 4 and 1, respectively, was $0.4 \%$. If the assumption $1=v_{1}+v_{2}+v_{3}$ leads to notable inaccuracies, partial molar volumes (see e.g. the review article by Imai (2007)) may be used to re-formulate it as $f\left(v_{1}, v_{2}, v_{3}\right)=v_{1}+v_{2}+v_{3}$, where $f\left(v_{1}, v_{2}, v_{3}\right)$ is an experimentally determined function of volume fractions $v_{1}, v_{2}$ and $v_{3}$. The disadvantage of this approach is that iterative image reconstruction algorithms that perform material decomposition for each voxel may suffer from the more time consuming solution of the resulting system of equations.

The L3 method is useful for decomposition of data measured by CT scanners as image reconstruction algorithms typically provide LACs and not MACs. General purpose particle transport codes using the Monte Carlo method require not only the elemental composition but also the mass density for each voxel. The derivation of mass density of the approximated mixture was based on the assumption that both masses and partial volumes of individual components are preserved in the mixture. If this is not the case, formula (A.10) can be replaced with an experimentally determined function $\rho\left(v_{1}, v_{2}, v_{3}\right)$.

Negative mass and volume fractions are nonphysical, nevertheless the meaning of quantities $W_{\left(n_{b} \times n_{v}\right)}$ and $V_{\left(n_{b} \times n_{v}\right)}$ in equations (A.3) and (A.4), respectively, can be generalized in the sense that a negative value means that corresponding amount of material should be removed from the mixture. For instance a tissue may contain a certain amount of calcium on the average. If calcium is selected as one of the base components then an excess or deficit of calcium will result in a positive or negative mass fraction, respectively. For the particle transport codes, the resulting elemental mass fractions should be positive. If they are not positive, different base materials must be used.

\subsection{Factors influencing accuracy of the three-material decomposition using $C T$ numbers from clinical DECT scanners}

Results presented in this paper are based on tissue compositions taken from literature to avoid bias and noise associated with CT numbers measured by contemporary clinical DECT scanners. The authors' experience is that a direct application of the L3 method on patient DECT data affected by image artifacts and noise typically results in large uncertainties and biases for the per-voxel volume fractions. Though the L3 method may provide good results in some cases (Liu et al 2009), its direct usage for radiation treatment planning is problematic as the current image reconstruction algorithms in clinical DECT scanners are not designed for quantitative CT. Work on the development of image reconstruction algorithms for quantitative DECT is in progress, see e.g. (Magnusson et al 2011). Optimal selection of base materials for the L3 method depends on the variability of elemental composition of tissues among patients. Some information 
on the variability is expressed in table 1 via three different values for the same tissue, e.g. liver 1,2, and 3. This variability, however, does not reflect pathological cases which are of interest in oncology. With the wider use of model based algorithms for brachytherapy, the need for quantitative assessment of the variability will raise. One approach is to analyze postmortem DECT scans. (Postmortem scans are used to lower statistical noise as there is no requirement for patient-dose reduction.) This work has been done e.g. at the Center for Medical Image Science and Visualization (CMIV), Linköping University, Sweden. This approach can further be combined with an elemental analysis of biopsy samples.

\section{Conclusion}

The decomposition of selected abdominal soft tissues to water, protein and lipid via the L3 method demonstrated that both mass energy absorption coefficients and mass attenuation coefficients of approximated mixtures did not differ by more than $2 \%$ from corresponding true values for photon energies larger than $10 \mathrm{keV}$. These differences were notably smaller than the ones for water as the transport and dose scoring medium (hereto used in treatment planning systems). The choice of the water, protein and lipid triplet resulted in negative elemental mass fractions for some analyzed tissues. As negative elemental mass fractions cannot be used in general purpose particle transport computer codes using the Monte Carlo method, other triplets should be used for the decomposition. The selection of suitable triplets, however, depends on variability of tissue composition among patients. More work is needed to estimate this variability and propose more sophisticated tissue decomposition schemes.

\section{Acknowledgments}

The authors thank the Center for Medical Image Science and Visualization (CMIV) at Linköping University, Sweden for access to the Siemens Somatom Definition DECT scanner. This work was supported by grants from the Swedish Cancer Society (Cancerfonden), contract number 100512.

\section{Appendix}

Appendix A.1. Matrix representation

For $n_{E}$ different photon energies $E_{1}, \ldots, E_{n_{E}},(1)$ can be written in matrix form as

$$
\left(\begin{array}{c}
\mu\left(E_{1}\right) / \rho \\
\vdots \\
\mu\left(E_{n_{E}}\right) / \rho
\end{array}\right)=\left(\begin{array}{ccc}
\mu_{1}^{\mathrm{b}}\left(E_{1}\right) / \rho_{1} & \ldots & \mu_{n_{b}}^{\mathrm{b}}\left(E_{1}\right) / \rho_{n_{b}} \\
\vdots & & \vdots \\
\mu_{1}^{\mathrm{b}}\left(E_{n_{E}}\right) / \rho_{1} & \ldots & \mu_{n_{b}}^{\mathrm{b}}\left(E_{n_{E}}\right) / \rho_{n_{b}}
\end{array}\right)\left(\begin{array}{c}
w_{1}^{\mathrm{b}} \\
\vdots \\
w_{n_{b}}^{\mathrm{b}}
\end{array}\right) \text {, }
$$

where $\mu\left(E_{i}\right) / \rho$ and $\mu_{j}^{\mathrm{b}}\left(E_{i}\right)$ are mass attenuation coefficients of the mixture and base material $j$, respectively, for photon energy $E_{i}$, and $w_{j}^{\mathrm{b}}$ is the mass fraction of the base 
material $j$. Using a notation with matrix dimensions as subscripts, (A.1) can be written as

$$
M_{\left(n_{E} \times 1\right)}=M_{\left(n_{E} \times n_{b}\right)}^{\mathrm{b}} W_{\left(n_{b} \times 1\right)}^{\mathrm{b}} .
$$

To simultaneously calculate MACs of $n_{v}$ different mixtures, the matrices $M_{\left(n_{E} \times 1\right)}$ and $W_{\left(n_{b} \times 1\right)}^{\mathrm{b}}$ in $(\mathrm{A} .2)$ can be extended by using $n_{v}$ columns of MACs and mass fractions, respectively, one column for each mixture:

$$
M_{\left(n_{E} \times n_{v}\right)}=M_{\left(n_{E} \times n_{b}\right)}^{\mathrm{b}} W_{\left(n_{b} \times n_{v}\right)} .
$$

This approach may speed up calculations on platforms that parallelize or vectorize matrix computations. Matrix formulation can also be used for the summation rule of LACs in (2). As in (A.3) the matrix multiplication can be written as

$$
L_{\left(n_{E} \times n_{v}\right)}=L_{\left(n_{E} \times n_{b}\right)}^{\mathrm{b}} V_{\left(n_{b} \times n_{v}\right)},
$$

where $L_{\left(n_{E} \times n_{v}\right)}$ and $L_{\left(n_{E} \times n_{b}\right)}^{\mathrm{b}}$ are matrices of LACs for the mixture and base materials, respectively, and $V_{\left(n_{b} \times n_{v}\right)}$ is the matrix of volume fractions.

In case of tissue decomposition, matrices $M_{\left(n_{E} \times 1\right)}$ and $M_{\left(n_{E} \times n_{b}\right)}^{\mathrm{b}}$ in (A.2) are known for $n_{E}$ effective energies corresponding to x-ray tube voltages of a CT scanner. In DECT, $n_{E}=2$. To obtain unknown mass fractions $W_{\left(n_{b} \times 1\right)}$, the normalization equation $1=w_{1}+\ldots+w_{n_{b}}$ must be added to the system of equations represented by (A.1). The resulting set of equations in a matrix form is then

$$
\left(\begin{array}{c}
M_{\left(n_{E} \times 1\right)} \\
1
\end{array}\right)=\left(\begin{array}{c}
M_{\left(n_{E} \times n_{b}\right)} \\
1_{\left(1 \times n_{b}\right)}
\end{array}\right) W_{\left(n_{b} \times 1\right)},
$$

where $1_{\left(1 \times n_{b}\right)}$ is a matrix with all elements equal to 1 . A set of $n_{v}$ voxels (an image) can be decomposed by solving the matrix equation

$$
\left(\begin{array}{c}
M_{\left(n_{E} \times n_{v}\right)} \\
1_{\left(1 \times n_{v}\right)}
\end{array}\right)=\left(\begin{array}{c}
M_{\left(n_{E} \times n_{b}\right)} \\
1_{\left(1 \times n_{b}\right)}
\end{array}\right) W_{\left(n_{b} \times n_{v}\right)} .
$$

Consequently, the mass fractions $W_{\left(n_{b} \times n_{v}\right)}$ can be calculated as

$$
W_{\left(n_{b} \times n_{v}\right)}=\left(\begin{array}{c}
M_{\left(n_{E} \times n_{b}\right)} \\
1_{\left(1 \times n_{b}\right)}
\end{array}\right)^{-1}\left(\begin{array}{c}
M_{\left(n_{E} \times n_{v}\right)} \\
1_{\left(1 \times n_{v}\right)}
\end{array}\right),
$$

where the superscript -1 stands for matrix inversion. A more efficient method of solving matrix equation (A.6) is for instance Gauss elimination, e.g. the routine solve() in the statistical program R ( R Development Core Team 2012). The same method used for the derivation of (A.7) can be used for the derivation of the matrix of volume fractions $V_{\left(n_{b} \times n_{v}\right)}$ from (A.4). The normalization condition is $1=v_{1}+\ldots+v_{n_{b}}$, and the resulting formula is

$$
V_{\left(n_{b} \times n_{v}\right)}=\left(\begin{array}{c}
L_{\left(n_{E} \times n_{b}\right)} \\
1_{\left(1 \times n_{b}\right)}
\end{array}\right)^{-1}\left(\begin{array}{c}
L_{\left(n_{E} \times n_{v}\right)} \\
1_{\left(1 \times n_{v}\right)}
\end{array}\right) .
$$


Appendix A.2. Mass density of a mixture

Let $m, V$, and $\rho$ be the mass, volume and mass density of a mixture. Let $m_{i}, V_{i}$, and $\rho_{i}$ be the same quantities of an $i$ th component of the mixture. Consider that the densities $\rho_{i}$ and mass fractions $w_{i}=m_{i} / m$ are known. Then the mass density of the mixture $\rho$ can be calculated as

$$
\rho=\left(\frac{V}{m}\right)^{-1}=\left(\frac{1}{m} \sum_{i} V_{i}\right)^{-1}=\left(\sum_{i} \frac{m_{i}}{m} \frac{V_{i}}{m_{i}}\right)^{-1}=\left(\sum_{i} w_{i} \rho^{-1}\right)^{-1} .
$$

Now consider that the densities $\rho_{i}$ and volume fractions $v_{i}=V_{i} / V$ are known. Then the mass density of the mixture $\rho$ can be calculated as

$$
\rho=\frac{m}{V}=\frac{1}{V} \sum_{i} m_{i}=\sum_{i} \frac{V_{i}}{V} \frac{m_{i}}{V_{i}}=\sum_{i} v_{i} \rho_{i}
$$

It is important to realize that the symbols $m_{i}$ and $V_{i}$ in equations (A.9) and (A.10) denote the mass and partial volume of the $i$ th component both inside (the ratios $m_{i} / m$ and $v_{i} / v$ ) and outside (the ratio $V_{i} / m_{i}$ ) the mixture. Thus the derivation of equations (A.9) and (A.10) requires that initial masses and partial volumes of individual components are preserved when these components are added to the mixture. While the conservation of mass is valid to a high degree of accuracy in chemical reactions, the conservation of partial volumes is only an approximation.

\section{References}

Aitchison J 1986 The statistical Analysis of Compositional Data (London: Chapman \& Hall) p 77

Alvarez R E and Macovski A 1976 Energy-selective reconstructions in X-ray computerised tomography Physics in Medicine and Biology 21 733-44

Attix F H 1986 Introduction to Radiological Physics and Radiation Dosimetry (New York: John Willey \& Sons) p 156

Bazalova M, Carrier J-F, Beaulieu L and Verhaegen F 2008a Dual-energy CT-based material extraction for tissue segmentation in Monte Carlo dose calculations Physics in Medicine and Biology 532439 56

Bazalova M, Carrier J-F, Beaulieu L and Verhaegen F 2008b Tissue segmentation in Monte Carlo treatment planning: A simulation study using dual-energy CT images Radiotherapy and Oncology $8693-8$

Beaulieu L, Carlsson Tedgren Å, Carrier J-F, Davis S D, Mourtada F, Rivard M J, Thomson R M, Verhaegen F, Wareing T A and Williamson J F (2012) Report of the Task Group 186 on Modelbased Dose Calculation Methods in Brachytherapy Beyond the TG-43 Formalism: Current Status and Recommendations for Clinical Implementation.Medical Physics 39 6208-6236.

Bornefalk H 2012 XCOM intrinsic dimensionality for low-Z elements at diagnostic energies Medical Physics 39654

Cullen D E, Hubbell J H and Kissel L 1997 EPDL97: the evaluated photon data library, '97 version, UCRL-50400 Vol. 6, Rev. 5, Lawrence Livermore National Laboratory, September 1997.

Goodsitt M M, Christodoulou E G and Larson S C 2011 Accuracies of the synthesized monochromatic $\mathrm{CT}$ numbers and effective atomic numbers obtained with a rapid $\mathrm{kVp}$ switching dual energy $\mathrm{CT}$ scanner. Med Phys 38 2222-32

Graser A, Johnson T R C, Bader M, Staehler M, Haseke N, Nikolaou K, Reiser M F, Stief C G and Becker C R 2008 Dual energy CT characterization of urinary calculi: Initial in vitro and clinical experience Investigative Radiology 431129 
Hubbell J H and Seltzer S M 2004 Tables of X-Ray Mass Attenuation Coefficients and Mass EnergyAbsorption Coefficients (version 1.4). [Online] Available: http://physics.nist.gov/xaamdi [2006, September 7]. National Institute of Standards and Technology, Gaithersburg, MD.

ICRP (International Commission on Radiological Protection) 1975 Report of the Task Group on Reference Man ICRP publication No. 23 (Oxford: Pergamon)

ICRU (International Commission on Radiation Units and Measurements) 1989 Tissue Substitutes in Radiation Dosimetry and Measurement ICRU Report 44 (Bethesda, MD: ICRU)

ICRU (International Commission on Radiation Units and Measurements) 1992 Photon, Electron, Proton and Neutron Interaction Data for Body Tissues ICRU Report 46 (Bethesda, MD: ICRU)

Imai T 2007 Molecular theory of partial molar volume and its applications to biomolecular systems Condensed Matter Physics 51 343-361

Landry G, Reniers B, Murrer L, Lutgens L, Bloemen-Van Gurp E, Pignol J-P, Keller B, Beaulieu L and Verhaegen F 2010 Sensitivity of low energy brachytherapy Monte Carlo dose calculations to uncertainties in human tissue composition Medical Physics 37 5188-98

Landry G, Granton P V, Reniers B, Öllers M C, Beaulieu L, Wildberger J E and Verhaegen F 2011a Simulation study on potential accuracy gains from dual energy CT tissue segmentation for lowenergy brachytherapy Monte Carlo dose calculations Physics in Medicine and Biology 566257

Landry G, Reniers B, Granton P V, van Rooijen B, Beaulieu L, Wildberger J E and Verhaegen F 2011b Extracting atomic numbers and electron densities from a dual source dual energy CT scanner: Experiments and a simulation model Radiother Oncol 100 375-9

Liu X, Yu L, Primak A N and McCollough C H 2009 Quantitative imaging of element composition and mass fraction using dual-energy CT: Three-material decomposition Medical Physics 361602

Magnusson M, Malusek A, Muhammad A and Alm Carlsson G 2011 Determination of Quantitative Tissue Composition by Iterative Reconstruction on 3D DECT Volumes. In: Proceedings of the 11th International Meeting on Fully Three-Dimensional Image Reconstruction in Radiology and Nuclear Medicine, July 11 - July 15, Potsdam, Germany (pages 120 - 123). Available at http://www.fully3d.org/

Petersilka M, Bruder H, Krauss B, Stierstorfer K and Flohr T G 2008 Technical principles of dual source CT European Journal of Radiology 68 362-8

Primak A N, Fletcher J G, Vrtiska T J, Dzyubak O P, Lieske J C, Jackson M E, Williams Jr J C and McCollough C H 2007 Noninvasive Differentiation of Uric Acid versus Non-Uric Acid Kidney Stones Using Dual-Energy CT Academic Radiology 14 1441-7

R Development Core Team 2012 R: A language and environment for statistical computing. R Foundation for Statistical Computing, Vienna, Austria. ISBN 3-900051-07-0, URL http://www.R-project.org/

Rivard M J, Venselaar J L and Beaulieu L 2009 The evolution of brachytherapy treatment planning Med. Phys. 36 2136-53

Scheffel H, Stolzmann P, Frauenfelder T, Schertler T, Desbiolles L, Leschka S, Marincek B and Alkadhi H 2007 Dual-Energy Contrast-Enhanced Computed Tomography for the Detection of Urinary Stone Disease Investigative Radiology December 200742 823-9

Schneider U, Pedroni E and Lomax A 1996 The calibration of CT Hounsfield units for radiotherapy treatment planning Physics in Medicine and Biology 41 111-24

Torikoshi M, Tsunoo T, Sasaki M, Endo M, Noda Y, Ohno Y, Kohno T, Hyodo K, Uesugi K and Yagi N 2003 Electron density measurement with dual-energy x-ray CT using synchrotron radiation Physics in Medicine and Biology 48 673-85

Woodard H Q and White D R 1986 The composition of body tissues Br J Radiol 59 1209-18 